\title{
Immunological Diagnostic Method
}

National Cancer Institute

\section{Source}

National Cancer Institute. Immunological Diagnostic Method. NCI Thesaurus. Code C18193.

Method used to identify disease using laboratory techniques involving interaction of antigens with specific antibodies. 